\title{
Application of bow-tie surface plasmon antenna to silicon on insulator nanowire photodiode for enhanced light absorption
}

\author{
Yash Sharma $^{1}$, Hiroaki Satoh ${ }^{2}$, and Hiroshi Inokawa ${ }^{2 a)}$ \\ ${ }^{1}$ Graduate School of Science and Technology, Shizuoka University, \\ 3-5-1 Johoku, Naka-ku, Hamamatsu 432-8011, Japan \\ ${ }^{2}$ Research Institute of Electronics, Shizuoka University, \\ 3-5-1 Johoku, Naka-ku, Hamamatsu 432-8011, Japan \\ a)inokawa.hiroshi@shizuoka.ac.jp
}

\begin{abstract}
Down scaling of photodetectors is one of the major approaches to enhance their performance in terms of operation speed, dark current and sensitivity to photogenerated carriers. In order to compensate for the drawback of the down scaling, i.e. the reduction of light absorption efficiency and light receiving area, this report introduces the bow-tie surface plasmon nanoantenna for silicon on insulator (SOI) nanowire photodiode and clarifies its spectroscopic response. The bow-tie structure has a light sensitive area in the scale of the wavelength, and resonantly enhances the electric field near the central gap resulting in increased generation of carriers in the silicon nanowire, which is experimentally verified and analyzed by electromagnetic simulation.
\end{abstract}

Keywords: bow-tie optical nanoantenna, silicon-on-insulator (SOI), surface plasmon, photodiode

Classification: Integrated optoelectronics

\section{References}

[1] S. Donati: Photodetectors (Prentice Hall, Upper Saddle River, 1999) 127.

[2] E. R. Fossum: "CMOS image sensors," IEEE Trans. Electron Devices 44 (1997) 1689 (DOI: 10.1109/16.628824).

[3] W. Du, et al.: "SOI metal-oxide-semiconductor field-effect transistor photon detector based on single-hole counting," Opt. Lett. 36 (2011) 2800 (DOI: 10. 1364/OL.36.002800).

[4] W. Du, et al.: "Single-photon detection by a simple silicon-on-insulator metaloxide-semiconductor field-effect transistor," Jpn. J. Appl. Phys. 51 (2012) 06FE01 (DOI: 10.7567/JJAP.51.06FE01).

[5] J.-P. Colinge: Silicon-on-Insulator Technology: Materials to VLSI (Kluwer Academic, 2004) 3rd ed. 2.

[6] S. Cristoloveanu: Electrical Characterization of Silicon-On-Insulator Materials and Devices (Kluwer Academic, Massachusetts, 1995).

[7] G. K. Celler, et al.: "Frontiers of silicon-on-insulator," J. Appl. Phys. 93 (2003) 
4955 (DOI: 10.1063/1.1558223).

[8] T. Ishi, et al:: "Si nano-photodiode with a surface plasmon antenna," Jpn. J. Appl. Phys. 44 (2005) L364 (DOI: 10.1143/JJAP.44.L364).

[9] D. Okamoto, et al:: "Numerical study of near-infrared photodetectors with surface-plasmon antenna for optical communication,” Jpn. J. Appl. Phys. 47 (2008) 2921 (DOI: 10.1143/JJAP.47.2921).

[10] J. Fujikata, et al.: "Waveguide-integrated si nano-photodiode with surfaceplasmon antenna and its application to on-chip optical clock distribution," Appl. Phys. Express 1 (2008) 022001 (DOI: 10.1143/APEX.1.022001).

[11] D. Okamoto, et al.: "InGaAs nano-photodiode enhanced using polarizationinsensitive surface-plasmon antennas," Jpn. J. Appl. Phys. 50 (2011) 120201 (DOI: 10.7567/JJAP.50.120201).

[12] H. Satoh, et al:: "Enhanced Visible light sensitivity by gold line-and-space grating gate electrode in thin silicon-on-insulator p-n junction photodiode," IEEE Trans. Electron Devices 60 (2013) 812 (DOI: 10.1109/TED.2012. 2235069).

[13] H. Satoh, et al:: "Material dependence of metal grating on SOI photodiode for enhanced quantum efficiency," IEEE Photonics Technol. Lett. 25 (2013) 1133 (DOI: 10.1109/LPT.2013.2260138).

[14] D. M. Schaadt, et al.: "Enhanced semiconductor optical absorption via surface plasmon excitation in metal nanoparticles," Appl. Phys. Lett. 86 (2005) 063106 (DOI: 10.1063/1.1855423).

[15] S. H. Lim, et al.: "Photocurrent spectroscopy of optical absorption enhancement in silicon photodiodes via scattering from surface plasmon polaritons in gold nanoparticles," J. Appl. Phys. 101 (2007) 104309 (DOI: 10.1063/ 1.2733649).

[16] S. P. Sundararajan, et al.: "Nanoparticle-induced enhancement and suppression of photocurrent in a silicon photodiode," Nano Lett. 8 (2008) 624 (DOI: 10. 1021/n1073030+).

[17] A. Ono, et al.: "Sensitivity improvement of silicon-on-insulator photodiode by gold nanoparticles with substrate bias control," Appl. Phys. Lett. 99 (2011) 062105 (DOI: $10.1063 / 1.3622650$ ).

[18] A. Ono, et al.: "Broadband absorption enhancement of thin SOI photodiode with high-density gold nanoparticles," Opt. Mater. Express 4 (2014) 725 (DOI: 10.1364/OME.4.000725).

[19] M. W. Knight, et al:: "Photodetection with active optical antennas," Science 332 (2011) 702 (DOI: 10.1126/science.1203056).

[20] D. P. Fromm, et al.: "Gap-dependent optical coupling of single "bowtie" nanoantennas resonant in the Visible," Nano Lett. 4 (2004) 957 (DOI: 10.1021/ nl049951r).

[21] A. Sundaramurthy, et al.: "Field enhancement and gap-dependent resonance in a system of two opposing tip-to-tip Au nanotriangles," Phys. Rev. B 72 (2005) 165409 (DOI: 10.1103/PhysRevB.72.165409).

[22] W. Ding, et al.: "Surface plasmon resonances in silver bowtie nanoantennas with varied bow angles," J. Appl. Phys. 108 (2010) 124314 (DOI: 10.1063/ $1.3524504)$.

[23] D. P. Fromm, et al.: "Exploring the chemical enhancement for surfaceenhanced Raman scattering with Au bowtie nanoantennas," J. Chem. Phys. 124 (2006) 061101 (DOI: 10.1063/1.2167649).

[24] J. Zhang, et al:: "Bowtie nanoantenna with single-digit nanometer gap for surface-enhanced raman scattering (SERS)," Plasmonics 10 (2015) 831 (DOI: 10.1007/s11468-014-9870-5). 
4952 (DOI: 10.1021/nl102963g).

[26] A. Kinkhabwala, et al:: "Large single-molecule fluorescence enhancements produced by a bowtie nanoantenna," Nat. Photonics 3 (2009) 654 (DOI: 10. 1038/nphoton.2009.187).

[27] H. Park, et al:: "Fabrication of metallic electrodes with nanometer separation by electromigration," Appl. Phys. Lett. 75 (1999) 301 (DOI: 10.1063/ 1.124354).

[28] C. Zhou, et al:: "Microfabrication of a mechanically controllable break junction in silicon," Appl. Phys. Lett. 67 (1995) 1160 (DOI: 10.1063/1.114994).

[29] L. Novotny: "Effective wavelength scaling for optical antennas," Phys. Rev. Lett. 98 (2007) 266802 (DOI: 10.1103/PhysRevLett.98.266802).

\section{Introduction}

In order to improve the speed performance of photodetectors, reduction of sizes is effective since carrier transit time and also the resistance-capacitance (RC) time constant can be reduced [1]. Size reduction also helps reducing the dark current (or dark count in case of single-photon detection) because of the less amount of generation centers included in the device. In addition, reduced capacitance leads to a larger charge-to-voltage conversion gain that is important for active pixel sensors [2] and some single-photon detectors [3, 4]. The effectiveness of the down scaling is enhanced especially when photodetectors are fabricated on silicon on insulator (SOI) substrates $[5,6,7]$ as the parasitic capacitance is further reduced by the lowpermittivity dielectric isolation, and the detector is perfectly separated from the thick substrate that can be a source of the dark current.

However, the downscaling of photodetectors has the serious drawback of the reduced light absorption efficiency and light receiving area. The introduction of an optical antenna that can concentrate light into a small volume is expected to alleviate the drawback and make the best use of the scaled-down photodetectors. Recently, the use of surface plasmon (SP) resonance is drawing attention to realize such an optical antenna. Ishi, et al. utilized a silver (Ag) concentric grating to generate SP and concentrate light on the small aperture at the center where a subwavelength silicon (Si) mesa photodiode (PD) was located [8]. Similar concentric and one-dimensional (line-and-space) gratings made of SP media, such as gold $(\mathrm{Au}), \mathrm{Ag}$ and aluminum (Al), were also applied to metal-semiconductor-metal (MSM) $[9,10,11]$ and metal-oxide-semiconductor (MOS) $[12,13]$ PDs although the propagating SP mode was not always employed, and the ability to expand the light receiving area was limited. Localized SP in Au nanoparticles and nanorods was reported to enhance the sensitivity of pn junction [14, 15, 16], MOS [17, 18] and Schottky [19] PDs, but they were not intended for scaled-down photodetectors with advantages mentioned above.

In this work, we report the application of the Au bow-tie SP antenna to nanowire SOI pn junction PD for the enhanced light absorption. The bow-tie structure consists of two triangles facing each other with a nanogap between them, where intense field is created [20, 21, 22] to enhance the light absorption in the nanowire placed nearby. The advantage of this structure is the small intersectional 
area between the antenna and the nanowire PD, which minimizes the increase in parasitic capacitance. To our knowledge, this is the first time for the bow-tie nanoantenna to be applied to the scaled-down PD, although it has been intensively researched for enhancing Raman scattering [23, 24, 25] and molecular fluorescence [26]. Here, we verify the enhancement of the light absorption in the nanowire PD experimentally, and analyze the antenna operation by electromagnetic simulation. Unique fabrication technique of nanogap in the bow tie by electromigration, which realizes single-digit nanometer gap, is also introduced for the first time.

\section{Device structure and fabrication}

\subsection{Device structure}

The structure of SOI nanowire PD with bow-tie nanoantenna is shown in Fig. 1(a). The channel of the PD connecting the cathode and anode, is in the form of nanowire of designed width $\left(W_{\mathrm{Si}}\right)$ of $150 \mathrm{~nm}$. As shown in Fig. 1(b), the nanoantenna is in the shape of a bow-tie.

The designed antenna length $\left(L_{\mathrm{ANT}}\right)$ ranges from 240 to $400 \mathrm{~nm}$, and designed channel length $\left(L_{\mathrm{C}}\right)$ of the SOI PD is from 40 to $72 \mathrm{~nm}$. Initially, the bow-tie is closed, but a nanogap can be created afterward by electromigration [27] at the junction as highlighted. Au is used as a material for bow-tie nanoantenna because of its inertness, i.e. its surface is more stable in atmospheric conditions compared to other SP media such as Ag. The bow-tie nanoantenna is designed as a halfwavelength antenna, i.e. $L_{\mathrm{ANT}}$ is a half of the SP propagation wavelength $\left(\lambda_{\mathrm{SP}}\right)$ as shown in the inset of Fig. 2(a).

Effective refractive index for the SP mode along $\mathrm{Au} /$ vacuum interface is given by

$$
n_{\mathrm{eff}}=\sqrt{\varepsilon_{1} \varepsilon_{2} /\left(\varepsilon_{1}+\varepsilon_{2}\right)},
$$

where, $\varepsilon_{1}$ and $\varepsilon_{2}$ are dielectric constants of vacuum and $\mathrm{Au}$, respectively. $\lambda_{\mathrm{SP}}$ along the $\mathrm{Au} /$ vacuum interface is given by

$$
\lambda_{\mathrm{SP}}=\frac{\lambda_{0}}{\operatorname{Re}\left\{n_{\mathrm{eff}}\right\}} .
$$

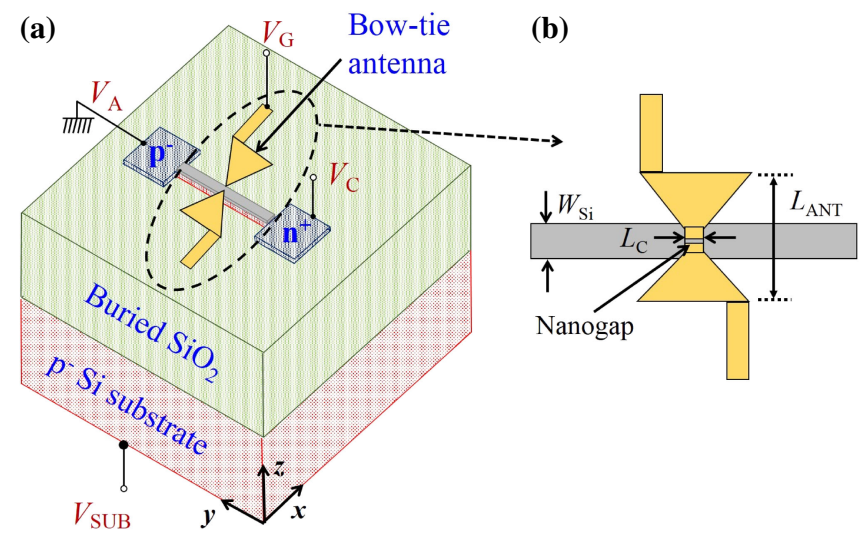


As seen from Fig. 2(a), $\lambda_{\mathrm{SP}}$ is not largely deviated from the wavelength in vacuum $\left(\lambda_{0}\right)$, and therefore $L_{\mathrm{ANT}}=240 \sim 400 \mathrm{~nm}$ is chosen to cover the visible wavelength. Attenuation of SP for half-SP-wavelength propagation is given by

$$
\text { Extinction ration }=1-e^{\frac{4 \pi I m\left|n_{\text {off }}\right|}{\lambda_{0}} \times \frac{\lambda_{\text {sp }}}{2}} \text {. }
$$

At $\lambda_{0}<550 \mathrm{~nm}$, interband transition occurs in Au, and SPs are absorbed as shown in Fig. 2(b), and as the result, antenna effect can be expected in the longer wavelength region.

\subsection{Device fabrication}

The SOI nanowire PD with bow-tie nanoantenna is fabricated on a commercial SOI wafer. The top Si layer and the substrate have p-type conduction, and the initial impurity (boron) concentration is $1 \times 10^{15} \mathrm{~cm}^{-3}$. Thickness of the top Si layer is $60 \mathrm{~nm}$, which is adjusted by thermal oxidation of the Si and removal of the oxide. The buried oxide (BOX) thickness is $400 \mathrm{~nm}$. Insulator between the bow-tie nanoantenna and $\mathrm{Si}$ nanowire is $\mathrm{SiO}_{2}$ with thickness of $20 \mathrm{~nm}$. The cathode of the PD is formed by thermal diffusion of phosphorus. Phospho-silicate spin-onglass (PSG) is spun on SOI, then phosphorus is diffused at $880^{\circ} \mathrm{C}$ for 20 minutes to achieve concentration $>2 \times 10^{19} \mathrm{~cm}^{-3}$. After removing PSG, the top silicon layer is patterned broadly by UV lithography to isolate PD from adjacent devices. The p-type Si region will form a channel connecting the cathode and anode of the PD. This channel is in the form of nanowires of various $W_{\mathrm{Si}}$ from 72 to $150 \mathrm{~nm}$, patterned using EBL system (JEOL JBX-6300SP) with EB energy of $100 \mathrm{keV}$ and dosage of $160 \mu \mathrm{C} / \mathrm{cm}^{2}$ for 100-nm-thick EB resist (Nippon Zeon ZEP-520A), and etched using $\mathrm{SF}_{6} / \mathrm{O}_{2}$-based reactive ion etching (SAMCO RIE-10NR). Nanowire will also act as the photosensitive region of the PD.

To enhance the photogeneration of carriers in the nanowire, Au nanoantennas in the shape of a bow tie with various designed $L_{\mathrm{ANT}}$ from 240 to $400 \mathrm{~nm}$ and $L_{\mathrm{c}}$ from 40 to $72 \mathrm{~nm}$ have been fabricated using resist lift-off technique. Firstly, the bow-tie shape is patterned using EBL with the same condition as that for delineating the top Si layer. After the exposure and development of EB resist, $5 \mathrm{~nm}$ of titanium (Ti) and $50 \mathrm{~nm}$ of $\mathrm{Au}$ are deposited by EB evaporation at a slow rate of $0.5 \mathrm{~nm} / \mathrm{s}$ to improve the surface morphology. Finally, the developed resist is lifted off by ultrasonic agitation to fabricate the pattern. Ti is inserted to improve adhesion strength between $\mathrm{Au}$ and $\mathrm{SiO}_{2}$, thereby improving the stability of the fabricated
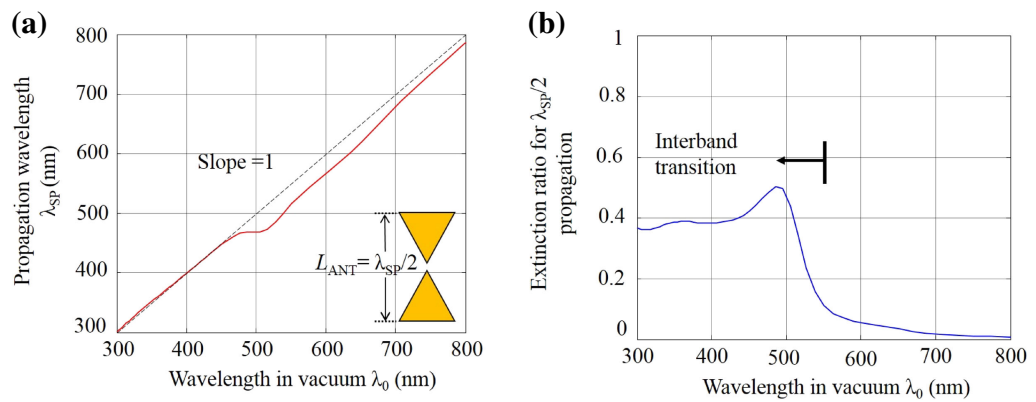

Fig. 2. (a) SP propagation wavelength vs. wavelength in vacuum, and (b) extinction ratio for half-SP-wavelength propagation. 


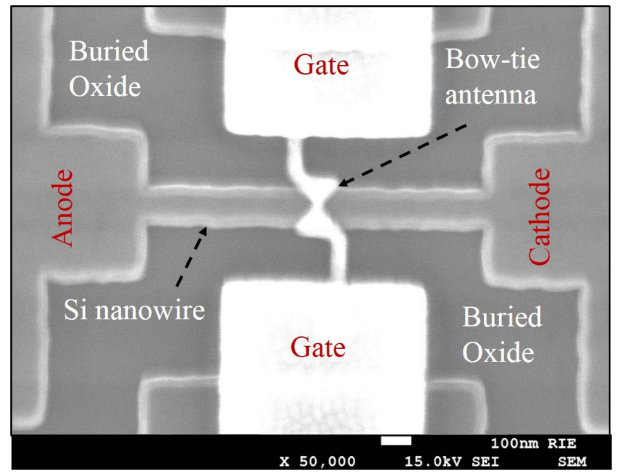

Fig. 3. FE-SEM enlarged view of the active area of Si nanowire PD with a bow-tie antenna.

nanostructure during lift-off. Fig. 3 shows field emission scanning electron microscopy (FE-SEM) image of the PD with $W_{\mathrm{Si}}, L_{\mathrm{ANT}}$ and $L_{\mathrm{C}}$ of 150, 240 and $40 \mathrm{~nm}$, respectively. External electrical biases are applied to contact pads, i.e. anode, cathode and two gates.

Since the bow tie is closed, a nanogap is created at the junction by flowing current and inducing electromigration [27]. The excited SP leads to the enhancement of the electric field near the nanogap [24]. The optical near field will generate the carriers within the depleted Si nanowire, thereby increasing the cathode current.

For optical measurement, the device is illuminated by the light through a monochromator with wavelength range from 400 to $800 \mathrm{~nm}$. The spectroscopic response of the SOI PD before and after formation of nanogap by breaking the bow-tie nanoantenna is measured at $T=300 \mathrm{~K}$ with biasing of $V_{\mathrm{C}}, V_{\mathrm{G}}$ and $V_{\mathrm{SUB}}$ at 1,0 and $-20 \mathrm{~V}$, respectively.

\subsection{Numerical simulation}

Using the three-dimensional (3D) finite difference time domain (FDTD) method, the near-field profile in the Au bow-tie antenna had been simulated. It is a reliable technique in solving Maxwell's equations in dispersive media. Si, $\mathrm{Au}$, and Ti were specified by frequency-dependent permittivity $\varepsilon(\omega)$ [12].

The FDTD simulations were carried out using the FullWAVE (RSOFT, Inc.). The quasi-plane wave irradiates the bowtie antenna on the SOI PD. The incident plane wave is linearly polarized light along the antenna length direction with wavelength $\lambda$ from 400 to $800 \mathrm{~nm}$, and propagates along the height direction of SOI PD. In this paper, the results in the cases with and without the gap are compared to clarify the field concentration effect of SP mode at the gap. The calculation domain with $1000 \times 1000 \times 630 \mathrm{~nm}^{3}$ (domain size in $x, y, z$ direction) was considered with a grid of $2 \mathrm{~nm}$, but the length of SOI channel and the planar size of BOX/Si substrate are infinite using perfectly matched layer (PML) as an absorbing boundary condition. For the calculation of the effective detector area, which will be discussed later, the absorption of light in the $\mathrm{Si}$ nanowire volume of $200 \times$ $150 \times 60 \mathrm{~nm}^{3}$ (length $\times$ width $\times$ thickness) is considered. 


\section{Results and discussion}

\subsection{Nanogap formation}

Fig. 4(a) shows the circuit diagram for breaking the bow-tie nanoantenna by controlled passage of current for formation of nanogap. The current flowing through the bow-tie nanoantenna is monitored. As shown in Fig. 4(b) the resistance at the onset of current flow (before breaking) is calculated to be around $60 \mathrm{G} \Omega$ at $40 \mathrm{mV}$. As the voltage across the antenna increases, the current also increases. At sufficiently high voltage, the current suddenly drops indicating the breaking of junction by electromigration. The resistance calculated after breaking the bow-tie nanoantenna is more than $2 \mathrm{~T} \Omega$.

The change in tunnel resistance reflects the change in the nanogap size. Quantitatively, the tunnel conductance $G_{\mathrm{t}}$ is exponentially dependent on the size of the nanogap $s$ formed at the center of the bow tie, and can be calculated by the equation $G_{\mathrm{t}}=G_{\mathrm{t} 0} \exp -\alpha \sqrt{ } \Phi_{S}$, where $\alpha$ is a characteristic length of tunneling $\left(=1.025 \AA^{-1} \mathrm{eV}^{-1 / 2}\right), \Phi$ is the work function of $\mathrm{Au}(=4 \mathrm{eV})$, and $G_{\mathrm{t} 0}$ is typically $0.8 \mathrm{~S}[28]$.

For tunnel resistances of $2 \mathrm{~T} \Omega$ after junction breaking, the nanogap size is estimated to be $1.4 \mathrm{~nm}$.

\subsection{Effective detector area}

Fig. 5 shows the effective detector area $\left(A_{\mathrm{Eff}}\right)$ of the SOI PD with respect to the wavelength for $L_{\mathrm{ANT}}=240 \mathrm{~nm}$ and $L_{\mathrm{C}}=40$ or $72 \mathrm{~nm}$, before and after breaking the bow-tie antenna to create nanogap. The $A_{\mathrm{Eff}}$ is defined by

$$
A_{E f f}=\frac{I_{P h}}{e} / \frac{P_{O p t}}{h v},
$$

where $I_{\mathrm{Ph}}, P_{\mathrm{Opt}}, e, h$ and $v$ are photocurrent, optical power per unit area, electron charge, Plank's constant and light frequency, respectively. The $A_{\mathrm{Eff}}$ is introduced instead of external quantum efficiency since the light receiving area is not clear.

The $A_{\text {Eff }}$ increases as the wavelength decreases, and shows a hump around the wavelength of $500 \mathrm{~nm}$. This is due to the high absorption coefficient of Si for the shorter wavelength region and the interferences in the multilayered structure [12].

In the wavelength range from 760 to $800 \mathrm{~nm}$, enhancement of the $A_{\text {Eff }}$ up to

(a)

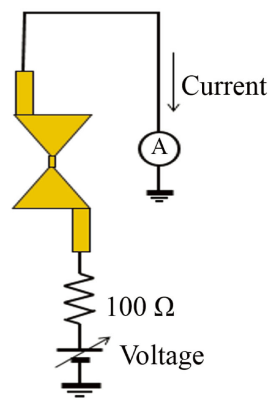

(b)

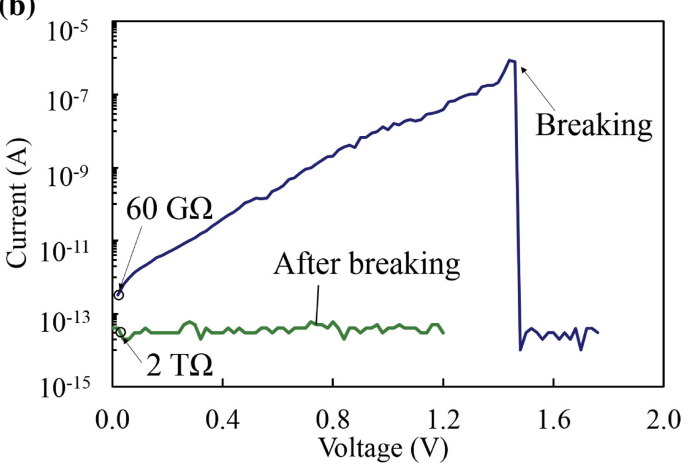




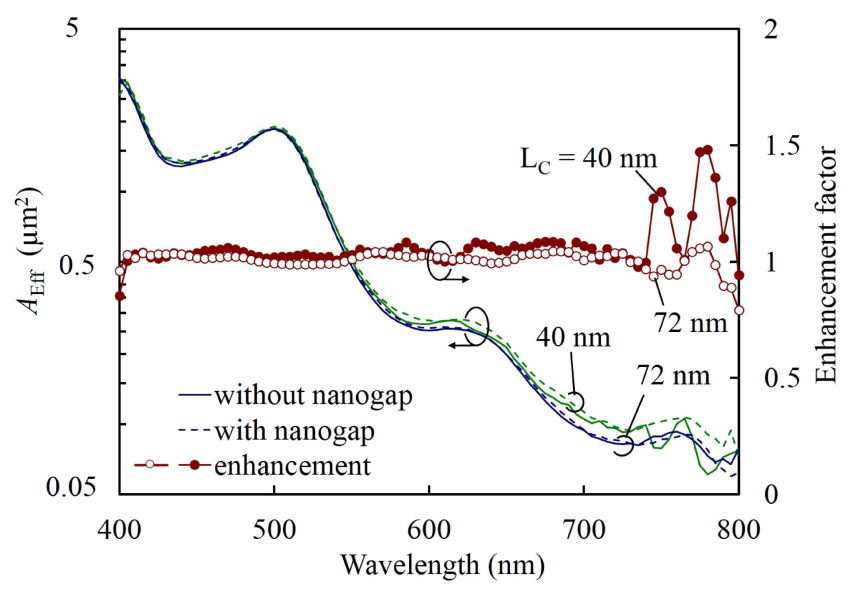

Fig. 5. Effective detector area $A_{\mathrm{Eff}}$ vs. wavelength for $L_{\mathrm{ANT}}=240 \mathrm{~nm}$ and $W_{\mathrm{Si}}=150 \mathrm{~nm}$.

$50 \%$ by the formation of nanogap is successfully observed for the $L_{\mathrm{C}}=40 \mathrm{~nm}$. The PD with $L_{\mathrm{C}}=72 \mathrm{~nm}$ does not show clear enhancement, probably because the concentration of the electromagnetic field is insufficient for the wider junction of the bow tie. The wavelength range for the enhancement is unexpectedly long for $L_{\mathrm{ANT}}=240 \mathrm{~nm}$ considering the SP propagation wavelength in Fig. 2(a). This might be attributed to the collective electron oscillation in the entire thickness of the thin Au layer [29].

\subsection{Numerical analysis}

Fig. 6 shows the simulated electric energy profile, $W_{\mathrm{e}}\left[\mathrm{J} / \mathrm{m}^{3}\right]$, of the Au bow-tie nanoantenna with $L_{\mathrm{ANT}}=240 \mathrm{~nm}$, with and without nanogap of $10 \mathrm{~nm}$ for the incident light with the power density of $1 \mathrm{~W} / \mu \mathrm{m}^{2}$ and the wavelength of $720 \mathrm{~nm}$. This wavelength is close to the SP resonance wavelength of the nanoantenna. The

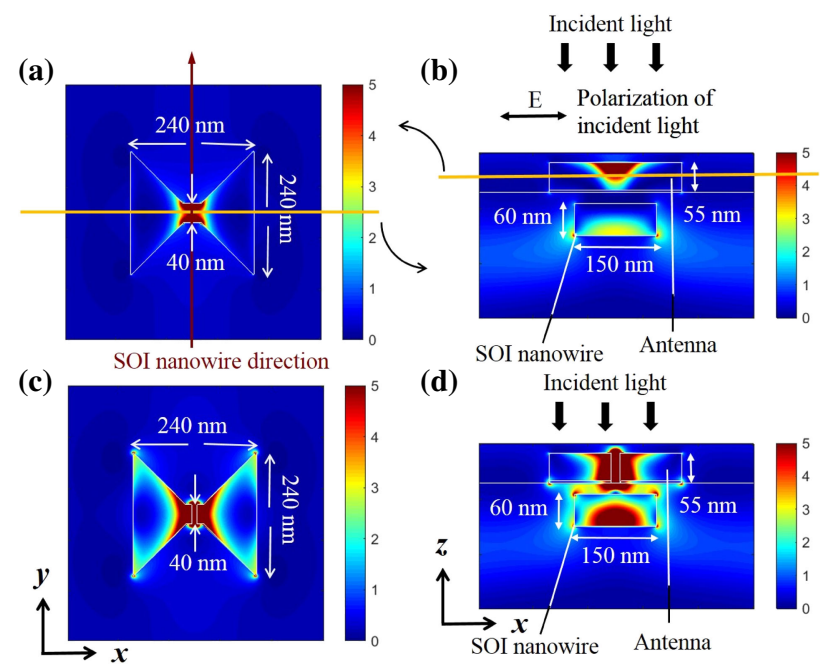

Fig. 6. Simulated electric energy profile, $W_{\mathrm{e}}=(1 / 2) \varepsilon|E|^{2}\left[\mathrm{~J} / \mathrm{m}^{3}\right]$, for Au bow-tie nanoantenna of $L_{\mathrm{ANT}}=240 \mathrm{~nm}, L_{\mathrm{C}}=40 \mathrm{~nm}$ and $W_{\mathrm{Si}}=150 \mathrm{~nm}$ illuminated by incident light with the wavelength of $720 \mathrm{~nm}$. (a) top and (b) cross-sectional views without nanogap, and (c) top and (d) cross-sectional views with nanogap of $10 \mathrm{~nm}$. 
gap size of $10 \mathrm{~nm}$ is selected to make the enhancement more visible since the range of the intense near field is in the order of the gap size, and the separation between the nanoantenna and the $\mathrm{Si}$ nanowire, i.e. the insulator $\left(\mathrm{SiO}_{2}\right)$ thickness, is $20 \mathrm{~nm}$.

As is observed in Fig. 6(a), electric energy is concentrated at the neck of the bow tie. By creating a nanogap, the density of electric energy is increased, and the intense electric energy is distributed inside the nanoantennas shown in Fig. 6(c). Comparing the cross-sectional views in Fig. 6(b) and (d) the intensity of electric field in the case with gap is much larger resulting in the enhancement of light absorption. Consequently, the cathode current would be increased.

Fig. 7 is the spectroscopic effective detector areas $A_{\text {Eff }}$ in the cases with and without 10-nm gap, and the enhancement factor by the nanogap. The enhancement factor larger than unity can be seen in the wavelength range from $660 \mathrm{~nm}$ to $780 \mathrm{~nm}$. The presence of nanogap of $10 \mathrm{~nm}$ in the neck of bow-tie nanoantenna increases the light absorption in the specified region in Si nanowire by more than $50 \%$ at wavelengths around $720 \mathrm{~nm}$. Similar characteristics have also been observed in the measured results in Fig. 5. In the experimental data, the $A_{\text {Eff }}$ is larger than that of the simulated one, and the enhancement factor is smaller. The former is probably caused by the contribution of the larger $\mathrm{Si}$ area compared to the one $\left(200 \times 150 \mathrm{~nm}^{2}\right)$ assumed in simulation. The latter is mainly due to the use of un-polarized light and the narrower gap in the experiment.

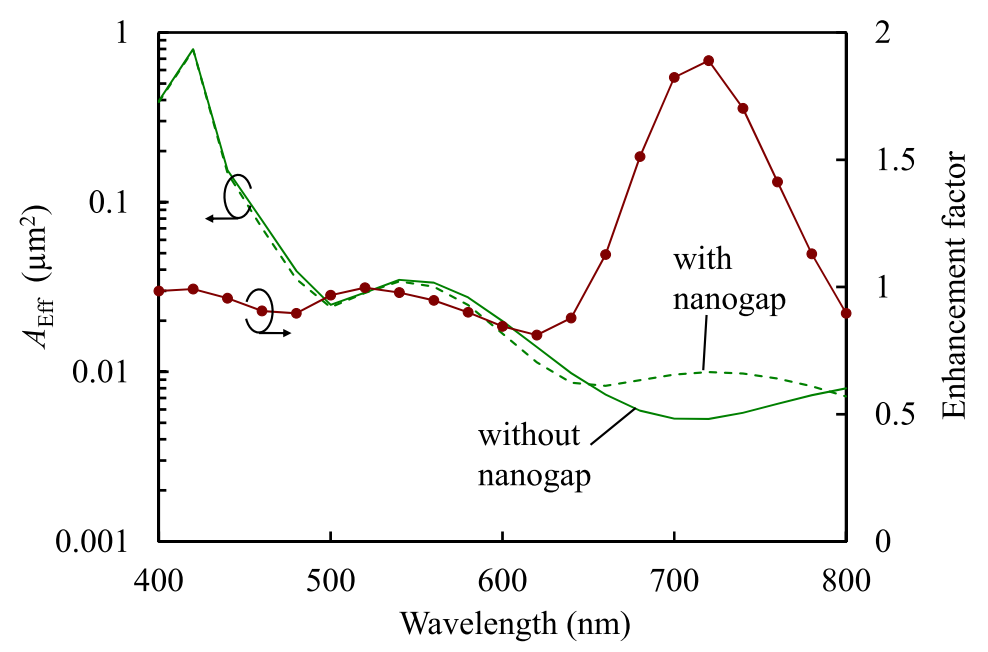

Fig. 7. Simulated effective detector area $A_{\mathrm{Eff}}$ vs. wavelength for $L_{\mathrm{ANT}}=240 \mathrm{~nm}$ and $W_{\mathrm{Si}}=150 \mathrm{~nm}$.

\section{Conclusions}

We fabricated the SOI nanowire PD with Au bow-tie nanoantenna having minimum feature size of $40 \mathrm{~nm}$ by EBL, and nanogap at the center with estimated size of $\sim 1.4 \mathrm{~nm}$ by electromigration. The $A_{\mathrm{Eff}}$ of the SOI nanowire PD were compared before and after the nanogap formation, and the enhancement up to $50 \%$ was successfully observed in the wavelength range from 760 to $800 \mathrm{~nm}$ for $L_{\mathrm{ANT}}=240 \mathrm{~nm}$. FDTD simulation was also carried out to confirm the concentration of the electric energy around the nanogap, and the enhanced $A_{\text {Eff }}$. The 
bow-tie nanoantenna with an electromigration-induced nanogap would be a unique measure to improve the efficiency of various nanometer-scale photonic devices.

\section{Acknowledgment}

This work was partly supported by JSPS KAKENHI Grant Number 25286068, the

Cooperative Research Project of Research Institute of Electronics, Shizuoka University, the Cooperative Research Project Program of RIEC, Tohoku University, and the Collaborative Research Program 2017 of Information Initiative Center, Hokkaido University. 